\title{
Expresiones de la violencia basada en género, en el marco del confinamiento por COVID-19
}

\author{
Gender Based Violence Manifestations on Lockdown Contexts by COVID-19
}

Nicolás Londoño Bernal ${ }^{1}$

\section{Resumen}

La violencia basada en género (VBG) es un reto actual para la consecución de la equidad de género y la garantía de una vida libre de violencia para las mujeres. Una vez que la emergencia por la expansión del virus COVID-19 inició, con las subsecuentes medidas de confinamiento adoptadas por distintos países, han aumentado los casos de VBG. La siguiente pesquisa lleva a cabo una revisión de textos provenientes de fuentes académicas, ONG y pronunciamientos oficiales acerca de la incidencia de las medidas de confinamiento sobre la VBG, especialmente para el caso iberoamericano. Desde una aproximación cualitativa, los resultados muestran expresiones, factores de riesgo y estrategias de mitigación para la prevención y atención de la VBG en el marco del confinamiento por COVID-19.

Palabras claves: violencia basada en género, VBG, confinamiento, COVID-19, violencia contra la mujer.

\section{Abstract}

Gender Based Violence (GBV) has been a major challenge in search for gender equality and the guarantee for a life without violence for women. Once COVID-19 emergency has begun, lockdown measures - adopted for many countries around the world - has revealed several increases in cases of GBV. The following research carries out a review at academics, ONGs, and formal pronouncements around the incidence of lockdown measures in GBV - especially for the Ibero-american context. Results indicate a qualitative approach to expressions, risk factors and mitigating measures for GBV at lockdown by COVID-19.

Keywords: Gender Based Violence, GBV, lockdown, COVID-19, violence against women.

1. Docente Universidad Santo Tomás, Colombia.

Correo electrónico: nicolaslondono@usantotomas.edu.co; nicolaslb01@gmail.com

ORCID: https://orcid.org/0000-0002-5621-4521

https://doi.org/10.22490/24629448.4194 


\section{Introducción}

La incidencia de violencia basada en género (VBG) es un factor estructural de inequidad en buena parte de las sociedades latinoamericanas y una de las afectaciones más graves para la salud de las mujeres y nińas de la región (1). En el marco de la actual pandemia por COVID-19 y las medidas de confinamiento adoptadas por distintos países, es necesario indagar por la relación entre el confinamiento y algunos factores de riesgo que aumentan tanto la gravedad como la incidencia de los distintos tipos de violencia de género y violencia contra la mujer (2-4).

El siguiente texto pretende compilar información acerca de factores de riesgo y medidas de mitigación de las expresiones de la violencia de género, mediante una revisión de literatura y textos relevantes en el marco del confinamiento por COVID-19, con el fin de brindar información actualizada en prevención de violencias basadas en género para potenciales actores involucrados. Específicamente, se retomará información de la región latinoamericana y de fuentes diversas como textos académicos, documentos de organizaciones internacionales y producción de organizaciones sociales de base.

En ese sentido, el artículo pretende ser un aporte para tomadores de decisiones, organizaciones civiles y cualquier persona víctima de violencia de género que busque rutas de acción frente a la violencia de género.

\section{Tipos de violencia y factores de riesgo}

Se procedió a la búsqueda de la información con una ecuación de búsqueda con palabras clave en referencia a la violencia de género y la COVID-19. Luego, se clasificó la información mediante una matriz para cada tipo de violencia de género con énfasis en los factores de riesgo y medidas de actuación para su prevención, atención y administración de justicia.

La violencia de género en el marco del confinamiento por COVID-19 ha sido invisibilizada dada la disminución general de delitos en los registros de sistemas de justicia estatales. El subregistro de violencia de género puede deberse a que el incremento de la VBG no se evidencia en las cifras de denuncia judicial, sino en las llamadas a servicios de atención especializada en violencia contra la mujer. Para el caso colombiano, las llamadas han aumentado en un $103 \%$; en México, cerca del $191 \%$; en Francia, un $30 \%$, y los aumentos tanto de llamadas como feminicidios se reportan en países como Argentina, Brasil, España, y el Reino Unido (5-15). En ese sentido, el hogar no representa siempre un lugar seguro. No se debe olvidar que, por ejemplo, en casos de feminicidio, los victimarios son la mayor de las veces las parejas actuales de las víctimas $(5,6)$.

Por ello, es necesario observar los factores de riesgo y recrudecimiento de los distintos tipos de VBG (16), así como algunas me- 
didas sugeridas o adoptadas por distintos países en la región.

Violencia sexual y física. El hecho de convivir en confinamiento con el maltratador constituye un factor de riesgo y reducción de acceso a la justicia, además de una continuación de ciclos de violencia $(2,13,14,17,18)$. Por ejemplo, para el caso del delito de feminicidio en Argentina $-y$ la tendencia es similar en el resto de la región- un $65 \%$ de las mujeres son asesinadas en su hogar y en un $60 \%$ de los casos el victimario es su pareja actual (12). Sumado a esto, se han señalado el cierre de programas de atención a derechos sexuales reproductivos, que pueden empeorar las afectaciones en salud a las víctimas de violencia sexual $(19,20)$, y la pérdida de motivación para realizar denuncias $(12,14)$, lo que, en general, hace más difícil identificar estas violencias convirtiéndolas en un hecho invisible (8). En particular, los hechos de violencia física y sexual en países con contextos de conflicto interno pueden incrementarse debido a una mayor inestabilidad social por la presencia de actores de guerra estatales (19).

Violencia psicológica. En este aspecto se resalta el aislamiento de redes de apoyo para identificar y sobrellevar hechos de violencia, lo que desemboca en sentimientos de soledad, distanciamiento emocional y corporal $(12,21)$. Por otra parte, se ha señalado una pérdida de autonomía debido a un aumento en trabajos de cuidado y el sometimiento a tiempos ajenos $(22,23)$. Además, una vez afectada la salud mental, se reporta acceso reducido a instancias de salud como psicología y psiquiatría. Todo esto se suma al factor estresante de salud mental que es el propio encierro (21,22,24-26).

Violencia económica y patrimonial. Se destaca la sobrecarga de trabajo doméstico sin retribución en cuidado y acompañamiento familiar con consecuentes dobles jornadas de trabajo $(4,8,19,22,27)$. Además, ha observado una precarización de trabajos de cuidado feminizados, como el de personal médico de primera línea, maestras o empleadas domésticas $(14,19,24,27,28)$. Por otro lado, se han suspendido cargos femeninos que se han considerado no esenciales en las empresas (8), renuncias forzadas $(22,28)$, y, a nivel regional, un mayor grado de informalidad en empleos para mujeres, lo que podría agravar su situación económica en la actual crisis $(11,19,20,29,30)$.

Violencia intrafamiliar. Es importante señalar un aumento en dinámicas de poder desigual, tales como restricción de movilidad hacia las mujeres $(8,11)$, y, en consecuencia, una menor capacidad de afrontamiento de la violencia (12). Existe un mayor riesgo de abuso sexual en niñas y niños y adolescentes (NNA), cuyos agresores son habitualmente familiares cercanos $(4,31)$. A ello se suma la desconexión con escenarios de identificación de abusos como la escuela (7). En 
este marco de violencia, puede presentarse miedo por parte de la madre a dejar el hogar debido a la situación de NNA y la falta de garantía en denuncias o restitución de derechos $(6,19)$.

Violencia simbólica e institucional. Se expresa en respuestas institucionales $y$ estatales, y se ha reportado el cierre o desfinanciación de programas de atención a VGB, y de medidas de protección como las cautelares o casas refugios (4, 11, 24). En ocasiones, se señalan deficiencias en implementar perspectiva de género en instituciones públicas o campañas que agudizan la violencia contra las mujeres y no reconocen la desigual división del trabajo doméstico (11).

Interseccionalidad. Para el caso de personas transgénero, el confinamiento ha empeorado las condiciones económicas de supervivencia, y retornar a sus hogares ha sido imposible debido a la discriminación y riesgo de salud que esto representa y las medidas discriminatorias de restricción de movilidad basadas en la expresión de género (32).

La precarización económica afecta también la vida de mujeres migrantes en países que han desfinanciado refugios (33), o en aquellos que ha suspendido la circulación, empobrecido sus dinámicas económicas y promovido campañas de odio; todo ello ha disparado el trabajo sexual forzado, la trata de personas y el matrimonio forzado $(19,24,28,34,35)$. Quizá una población fuertemente afectada son los NNA víctimas de violencia que quedan confinados con sus agresores, sin posibilidad de detección temprana del abuso o con grados de desescolarización cuando se obliga a niñas a participar de la demanda de cuidado del hogar (23).

Por último, des importante continuar con los servicios de salud sexual y reproductiva (por ejemplo, la interrupción del embarazo en las condicionales que cada país permite) $(24,30,36,37)$.

Estrategias de atención y prevención. Entre las estrategias de éxito reportadas por la literatura se destacan: articulación y apoyo con organizaciones de mujeres y ONG internacionales $(5,19,20,38)$; alianzas e innovaciones para espacios, servicios, y redes comunitarias que ofrecen estas organizaciones $(6,11)$; nuevos canales y protocolos virtuales para las denuncias de género con nuevas medidas de protección en confinamiento $(5,12,19,20)$, como las medidas de alerta $(13,17)$ o asignación de custodias (39), y continuación en la prestación de servicios de salud y atención psicológica y psiquiátrica $(11,17,27,40,41)$. Por último, se anima la creación de programas para personas cuidadoras de NNA, adultos mayores o personas con algún tipo de discapacidad (38), y de campañas dirigidas a mujeres y hombres que promuevan una distribución equitativa de estas actividades en el hogar $(16,19)$. Una mención especial refiere la importancia de generar datos desagregados por género durante la pandemia 
para lograr acciones eficaces y pertinentes $(19,24,38)$.

\section{Conclusión}

Las medidas de confinamiento han recreado, agravado e intensificado las violencias de género y violencia contra la mujer en diferentes países de Iberoamérica. Las fuentes permiten comprender una dinámica regional de violencia en el ámbito doméstico que es estructural, anterior al confinamiento, pero que adquiere invisibilidad y un mayor grado de dificultad en su atención por las distancias entre las víctimas y las posibles redes y rutas de prevención y atención de VBG. En este sentido, se requieren acciones novedosas coordinadas con organizaciones de base y entidades estatales para garantizar rutas de justicia y restauración de derechos a las víctimas, así como acciones de prevención basadas en evidencia dirigidas a hombres y mujeres que promuevan una cultura de la equidad de género.

\section{Referencias}

1. Viveros M, Arango LG. El género: una categoría útil para las ciencias sociales [Internet]. Bogotá D.C.: Universidad Nacional de Colombia, Facultad de Ciencias, Escuela de Estudios de Género, Grupo Interdisciplinario de Estudios de Género (GIEG); 2014. Disponible en: http://www.digitaliapublishing.com/a/62149/

2. Hall BJ, Tucker JD. Surviving in place: The coronavirus domestic violence syndemic. Asian J Psychiatr.
2020;53:102179. DOI: https://doi.org/10.1016/j. ajp.2020.102179

3. Mazza M, Marano G, Lai C, Janiri L, Sani G. Danger in danger: Interpersonal violence during COVID-19 quarantine. Psychiatry Res. 2020;289:113046. DOI: https://doi.org/10.1016/j.psychres.2020.113046.

4. John N, Casey SE, Carino G, McGovern T. Lessons Never Learned: Crisis and gender-based violence. Dev World Bioeth. 2020;20(2):65-8.

5. ONU. La ONU y Argentina luchan con la otra pandemia del coronavirus, la violencia de género [Internet]. Noticias ONU. 2020 [cited 2020 Jul 1]. Disponible en: https://news.un.org/es/ story/2020/04/1473082

6. Ayuda en acción. Coronavirus y violencia de género: un binomio peligroso [Internet]. Blog Web. 2020 [cited 2020 Jul 1]. Disponible en: https://ayudaenaccion.org/ong/blog/mujer/coronavirus-violencia-genero/

7. Feminicidios.NET. Feminicidios y otros asesinatos de mujeres cometidos en 2020 [Internet]. 2020 [cited 2020 Jul 1]. Disponible en: https://feminicidio. net/articulo/feminicidios-y-otros-asesinatos-mujeres-cometidos-2020

8. Buchholz Y. COVID-19: Incremento de la violencia de género [Internet]. 2020. Disponible en: https:// www.investigacionyciencia.es/noticias/covid-19-incremento-de-la-violencia-de-gnero-18549

9. CIDE. Violencia de Género en tiempos de COVID-19 [Internet]. Divulgación científica CIDE Covid 19. 2020 [cited 2020 Jul 1]. Disponible en: https://www.cide.edu/coronavirus/2020/05/11/violencia-de-genero-en-tiempos-de-covid-19/

10. Dávila V. Golpeadores miserables. Semana [Internet]. 2020 Apr 10; Disponible en: https://www. semana.com/opinion/articulo/covid-19-y-violencia-contra-la-mujer-golpeadores-miserables-por-vicky-davila/ 662592

11. Ruiz Hurtado A. En la cuarentena, la casa no es un lugar seguro para las mujeres. El Tiempo [Internet]. 
2020 Apr 18; Disponible en: https://www.eltiempo. $\mathrm{com} / \mathrm{mundo} / \mathrm{mas}$-regiones/aumentan-las-denuncias-de-violencia-de-genero-durante-los-confinamientos-por-el-coronavirus- 485864

12. Carrasco, Liliana; Martínez M. Riesgos inminentes, cuerpos descorporizados, silencios que gritan, luchas colectivas o muerte: efectos de la pandemia COVID-19 en la configuración de las violencias contra las mujeres. RED Soc [Internet]. 2020;07(02):12.

13. Sordo, Tania; Laporta E. El feminicidio en Espańa: Entre el rechazo conceptual y las resistencias político-jurídicas. Iberoamérica Soc Rev Estud Soc [Internet]. 2020;14(8):28-49. Disponible en: https:// iberoamericasocial.com/ojs/index.php/IS/article/ view/432

14. Bradbury-Jones C, Isham L. The pandemic paradox: The consequences of COVID-19 on domestic violence. J Clin Nurs. 2020;29(13-14):2047-9.

15. Marques ES, de Moraes CL, Hasselmann MH, Deslandes SF, Reichenheim ME. Violence against women, children, and adolescents during the COVID-19 pandemic: Overview, contributing factors, and mitigating measures. Cad Saude Publica. 2020;36(4). DOI: https://doi.org/10.1590/0102-311x00074420.

16. van Gelder N, Peterman A, Potts A, O'Donnell M, Thompson K, Shah N, et al. COVID-19: Reducing the risk of infection might increase the risk of intimate partner violence. EClinicalMedicine. 2020;21:100348. DOI: https://doi.org/10.1016/j. eclinm.2020.100348

17. Gallegos M, Zalaquett C, Sánchez SEL, Mazo-Zea R, Ortiz-Torres B, Penagos-Corzo JC, et al. Coping with the Coronavirus (COVID-19) pandemic in the Americas: Recommendations and guidelines for mental health Interam J Psychol. 2020;54(1).

18. Kofman YB, Garfin DR. Home Is Not Always a Haven: The Domestic Violence Crisis Amid the COVID-19 Pandemic. Psychol Trauma Theory, Res Pract Policy. 2020;12(S1):S199-S201. DOI: https:// doi.org/10.1037/tra0000866
19. Mujeres O. COVID-19 en América Latina y el Caribe: cómo incorporar a las mujeres y la igualdad de género en la gestión de la respuesta a la crisis [Internet]. Panamá; 2020. Disponible en: https:// www2.unwomen.org/-/media/field office americas/ documentos/publicaciones/2020/03/briefing coronavirusv1 117032020.pdf?la=es\&vs=930

20. Woman U. COVID-19 and Ending Violence Against Women and Girls [Internet]. 2020 [cited 2020 Jul 1]. p. 1-10. Disponible en: https://www. unwomen.org/-/media/headquarters/attachments/ sections/library/publications/2020/issue-brief-covid-19-and-ending-violence-against-women-andgirls-en.pdf?la=en \&vs=5006

21. Galea S, Merchant RM, Lurie N. The Mental Health Consequences of COVID-19 and Physical Distancing: The Need for Prevention and Early Intervention. JAMA Intern Med. 2020;180(6):817-8.

22. Fernández AR. El tiempo de las mujeres: trabajo y malestar femenino en tiempos de pandemia. Rev Reflexiones [Internet]. 2020 Jun 4;99(2 SE-Dossier especial 99 (2) 2020). Disponible en: https://revistas. ucr.ac.cr/index.php/reflexiones/article/view/42150

23. Formichella MM, Krüger NS. Pandemia y brechas educativas: reflexiones desde la Economía de la Educación. 2020 [cited 2020 Jul 18]; Disponible en: http://ri.conicet.gov.ar/handle/11336/109085

24. ONU Mujeres. COVID-19 y su impacto en la violencia contra las mujeres y nińas [Internet]. 2020 [cited 2020 Jul 1]. p. 1-4. Disponible en: https:// www2.unwomen.org/-/media/field office mexico/ documentos/publicaciones/2020/abril 2020/covid19_violenciamujeresninas_generalabril2020.pdf? $1 \mathrm{a}=\mathrm{es} \& \mathrm{vs}=2457$

25. Pikielny A. Es un equívoco pensar que la distancia física no es una distancia social. Entrevista a Rita Segato. In: Manrique Guzmán A, editor. El coronavirus y su impacto en la sociedad actual y futura. Lima, Perú: Colegio de Sociólogos del Perú; 2020. p. 387-93. 
26. Mackolil J, Mackolil J. Addressing psychosocial problems associated with the COVID-19 lockdown. Asian J Psychiatr. 2020;51:102156. DOI: https:// doi.org/10.1016/j.ajp.2020.102156

27. Sanahuja JA. COVID-19: riesgo, pandemia y crisis de gobernanza global. Anu CEIPAZ 2019-2020 Riesgos Glob y Multilater el impacto la COVID-19 [Internet]. 2020;27-54. Disponible en: http://www. ceipaz.org/images/contenido/2020-ANUARIO COMPLETO.pdf

28. Asociación con la A C. Educación, género y coronavirus. Asoc con la a [Internet]. 2012 May 25 [cited 2020 Jul 18];(69):1-5. Disponible en: http://repositori.uji.es/xmlui/handle/10234/188634

29. Bonaglia, Federico; Nieto-Parra, Sebastián; Vázquez-Zamora J. Una mirada al futuro post-covid-19: hacia un nuevo pacto social en América Latina y el Caribe [Internet]. Análisis Carolina. 2020. p. 1-15. Disponible en: https://www.fundacioncarolina.es/ wp-content/uploads/2020/04/AC-21.-2020.pdf

30. Gausman J, Langer A. Sex and Gender Disparities in the COVID-19 Pandemic. J Women's Heal. 2020;29(4):465-6.

31. Ghosh R, Dubey MJ, Chatterjee S, Dubey S. Impact of COVID -19 on children: special focus on the psychosocial aspect. Minerva Pediatr. 2020;72(3):22635 .

32. Radi, Blas; Losada Castilla C. Transmasculinidades y Covid-19 en América Latina y el caribe [Internet]. 2020. Disponible en: https://www.ilga-lac.org/coronapapers.pdf

33. Reporte situacional: migración de tránsito en México durante la pandemia de COVID-19 [Internet]. Ciudad de México; 2020. Disponible en: https://bit. ly/2Fusq3x

34. ACNUR. La pandemia del coronavirus aumenta el riesgo de violencia de género hacia mujeres y niñas desplazadas y apátridas [Internet]. 2020 [cited 2020 Jul 1]. Disponible en: https://www.acnur.org/noticias/press/2020/4/5e9d5f5d4/la-pandemia-del-co- ronavirus-aumenta-el-riesgo-de-violencia-de-genero-hacia.html

35. Roesch E, Amin A, Gupta J, García-Moreno C. Violence against women during covid-19 pandemic restrictions. BMJ. 2020;369.

36. Tang K, Gaoshan J, Ahonsi B. Sexual and reproductive health (SRH): A key issue in the emergency response to the coronavirus disease (COVID-19) outbreak. Reprod Health. 2020;17(1).

37. Viveiros N, Bonomi AE. Novel Coronavirus (COVID-19): Violence, Reproductive Rights and Related Health Risks for Women, Opportunities for Practice Innovation. J Fam Violence. 2020: 1-5. DOI: https://doi.org/10.1007/s10896-020-00169-x

38. Bárcena A. Coyuntura, escenarios y proyecciones hacia 2030 ante la presente crisis de Covid-19 [Internet]. CEPAL - Observatorio COVID - 19 en América Latina y el Caribe. 2020 [cited 2020 Jul 1]. Disponible en: http://www.cietalbidt.com/archivos/ Informe/Alicia Barcena CEPAL - PPT Impacto COVID-19 en ALC - 3-Abr-2020.pdf.pdf

39. Cardozo Ramírez M. ANÁLISIS DEL RÉGIMEN DELA GUARDA Y CUSTODIACOMPARTIDADE LOS HIJOS MENORES [Internet]. Universidad de la Laguna; 2020. Disponible en: https://riull. ull.es/xmlui/bitstream/handle/915/19652/Analisis de la guarda y custodia compartida de los hijos menores $\cdot$ pdf? sequence $=1$

40. Monereo Pérez JL, Rodríguez Iniesta G. La protección social en la emergencia. Entre el ensayo, precipitación y búsqueda de soluciones en tiempos de incertidumbre. Rev Derecho la Segur Soc Laborum 2020;2(23):11-53. Disponible en: https://revista.laborum.es/index.php/revsegsoc/article/view/408

41. Gulati G, Kelly BD. Domestic violence against women and the COVID-19 pandemic: What is the role of psychiatry? Int J Law Psychiatry. 2020;71:101594. DOI: https://doi.org/10.1016/j.ijlp.2020.101594 